University of Wollongong

Research Online

Faculty of Informatics - Papers (Archive)

Faculty of Engineering and Information

Sciences

$1-1-2008$

\title{
Multipath diversity of precoded OFDM with linear equalization
}

Xiaojing Huang

University of Wollongong, huang@uow.edu.au

Follow this and additional works at: https://ro.uow.edu.au/infopapers

Part of the Physical Sciences and Mathematics Commons

\section{Recommended Citation}

Huang, Xiaojing: Multipath diversity of precoded OFDM with linear equalization 2008, 1307-1311.

https://ro.uow.edu.au/infopapers/1433

Research Online is the open access institutional repository for the University of Wollongong. For further information contact the UOW Library: research-pubs@uow.edu.au 


\title{
Multipath diversity of precoded OFDM with linear equalization
}

\begin{abstract}
This paper settles a controversy over the multipath diversity performance of the precoded orthogonal frequency division multiplexing system with the linear equalization. Through the asymptotical analysis of the bit error rates with extreme system parameters, a comprehensive understanding of the linear equalization's behavior in the frequency-selective multipath fading channels is gained. Compared with the optimum maximum-likelihood detection, the diversity performance of the linear equalization can be well described by an asymptotical signal-to-noise ratio (SNR) degradation, which reveals that the linear equalization can achieve the maximum multipath diversity within an operational SNR range but will lose diversity advantage as SNR increases.
\end{abstract}

\section{Keywords}

Multipath, diversity, precoded, OFDM, linear, equalization

Disciplines

Physical Sciences and Mathematics

\section{Publication Details}

X. Huang, "Multipath diversity of precoded OFDM with linear equalization," in IEEE International Conference on Communications, 2008, pp. 1307-1311. 


\title{
Multipath Diversity of Precoded OFDM with Linear Equalization
}

\author{
Xiaojing Huang \\ School of Electrical, Computer and Telecommunications Engineering \\ University of Wollongong \\ Wollongong, Australia
}

\begin{abstract}
This paper settles a controversy over the multipath diversity performance of the precoded orthogonal frequency division multiplexing system with the linear equalization. Through the asymptotical analysis of the bit error rates with extreme system parameters, a comprehensive understanding of the linear equalization's behavior in the frequency-selective multipath fading channels is gained. Compared with the optimum maximum-likelihood detection, the diversity performance of the linear equalization can be well described by an asymptotical signal-to-noise ratio (SNR) degradation, which reveals that the linear equalization can achieve the maximum multipath diversity within an operational SNR range but will lose diversity advantage as SNR increases.
\end{abstract}

Keywords-orthogonal frequency division multiplexing (OFDM); multipath diversity; linear equlaization.

\section{INTRODUCTION}

Orthogonal frequency division multiplexing (OFDM) provides an efficient means to mitigate the intersymbol interference (ISI) caused by the channel multipath spread and enjoys the simple frequency domain channel equalization via fast Fourier transform (FFT). However, in addition to the large peak-to-average power ratio (PAPR) and the necessity for complicated frequency synchronization, conventional OFDM systems suffer from a major disadvantage of poor diversity performance in frequency-selective fading channels. Channel coding has been traditionally used to improve the diversity across frequency and time, and recently linear precoding and block spreading are introduced for OFDM systems to gain frequency diversity [1-4].

Though there are some variations in performing precoding, the precoded OFDM systems share the same principle of applying a unitary matrix to a group of data symbols before subcarrier mapping. Since the precoded data symbol modulated on a subcarrier is now a linear combination of the original data symbols, if any subcarrier experiences a deep fade after transmitting over a frequency-selective multipath channel, the original data symbols can be still recovered from other received subcarriers, so that the system performance is improved due to the increased diversity order.

Several authors have proved, using a pairwise error probability (PEP) analysis, that the precoded OFDM can achieve the maximum diversity advantage with the maximumlikelihood (ML) detection if the precoding data group size is larger than or equal to the channel diversity order [1,2]. Since

This research is supported by the Australian Research Council Discovery Project DP0558405. the ML detection is highly computationally complicated, especially when the data group size is large, a linear equalization, such as the minimum mean squared error (MMSE) equalization and the zero-forcing (ZF) equalization, followed by a hard decision is preferable in practice. However, regarding the performance of the precoded OFDM with linear equalization, there is a controversy found in the literature. Tepedelenlioglu claims in [3], based on the PEP analysis, that a precoded OFDM system can always achieve maximum multipath diversity through linear equalization, whereas McCloud concludes in [4], by asymptotical error performance analysis for only ZF equalization, that the linear equalization does not gain any diversity advantage for block spread OFDM (which is also a precoded OFDM).

In this paper, a different asymptotical approach is taken to investigate the diversity performance of the linear equalization for the precoded OFDM. We first analyze the bit error rate (BER) of the linear equalization under two system parameters, i.e., the data group size and the channel diversity order, assuming a quadrature phase shift keying (QPSK) subcarrier mapping. Then, we derive the asymptotical performance by putting the two parameters into different extreme conditions. We also determine a performance lower bound of the ML detection, so that the performance of the linear equalization can be compared, and the diversity advantage can be assessed. After defining an asymptotical SNR degradation, a comprehensive understanding of the linear equalization's behavior is gained, and thus the controversy over the diversity performance of the linear equalization is settled.

The rest of the paper is organized as follows. In Section II, the precoded OFDM system models are presented. In Section III, the BER of the linear equalization is formulated as a function of the data group size and the multipath diversity order. Section IV is devoted to the derivation of the closedform asymptotical BER of the linear equalization. Section V provides the evaluation results of the derived asymptotical BER and defines the asymptotical SNR degradation. Finally, conclusions are drawn in Section VI.

\section{SYSTEM AND CHANNEL MODELS}

The precoded OFDM transmitter baseband model is shown in Fig. 1 (a), where a block of $M N$ input data symbols ( $M$ and $N$ are chosen as integer powers of 2 for convenience) is denoted as a sequence $x[i], i=0,1, \cdots, M N-1$, or vector $\mathbf{x}$. 
Before precoding, $x[i]$ is firstly divided, through serial-toparallel conversion (S/P), into $N$ groups of size $M$ with the $n$th group, $n=0,1, \cdots, N-1$, being denoted as a vector

$$
\mathbf{x}_{n}=(x[n M], x[n M+1], \cdots, x[n M+M-1])^{T}
$$

where $(\cdot)^{T}$ denotes the matrix transposition.

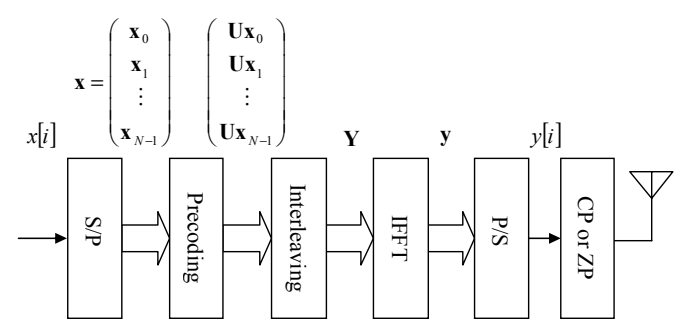

(a)

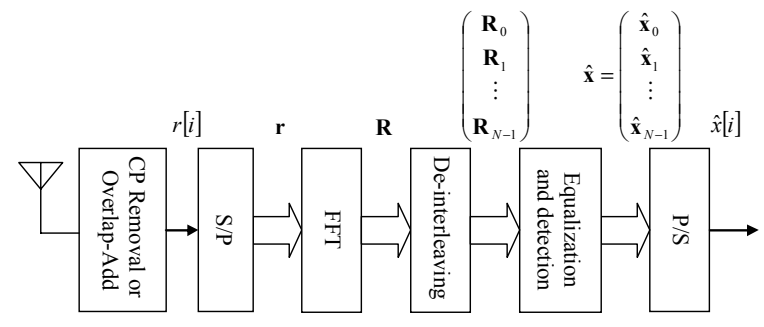

(b)

Fig. 1. Precoded OFDM system models: (a) transmitter and (b) receiver.

The precoding process is to apply an $M \times M$ unitary matrix $\mathbf{U}$, which satisfies the property $\mathbf{U} \mathbf{U}^{\prime}=\mathbf{U}^{\prime} \mathbf{U}=\mathbf{I}$, where $(.)^{\prime}$ denotes the matrix transposition and complex-conjugation operation and $\mathbf{I}$ is the identity matrix of order $M$, to each vector $\mathbf{x}_{n}$ to produce a precoded vector $\mathbf{U} \mathbf{x}_{n}$, and thus each element in the precoded vector is a linear combination of the symbols in vector $\mathbf{x}_{n}$. To better exploit frequency diversity, the precoded symbols are preferably mapped onto subcarriers equally spaced across the transmitted bandwidth [2]. This mapping can be implemented by performing a block interleaving operation among the $N$ precoded vectors and then taking an IFFT of length $M N$ on the interleaved vector $\mathbf{Y}$. The resulting vector is denoted as $\mathbf{y}$.

After parallel-to-serial conversion $(\mathrm{P} / \mathrm{S}), \mathbf{y}$ is converted into a time domain sequence $y[i], i=0,1, \cdots, M N-1$. To form a precoded OFDM symbol, either a cyclic prefix (CP) or a zeropadded (ZP) suffix of sufficient length (longer than the maximum channel multipath delay $L$ in samples) need to be added to $y[i]$ to avoid interference between adjacent precoded OFDM symbols and turn the linear convolution of the transmitted signal with the channel impulse response into a circular one.

The precoded OFDM signal is then transmitted over a frequency-selective multipath fading channel described by the discrete channel impulse response $h[i], i=0,1, \cdots, L-1$, and received at the receiver baseband. By removing the $\mathrm{CP}$ or performing an overlap-add operation, $M N$-point received samples $r[i], i=0,1, \cdots, M N-1$, are produced, which are represented as vector $\mathbf{r}$ after $\mathrm{S} / \mathrm{P}$, and further transformed into frequency domain by FFT to yield a vector $\mathbf{R}$. After deinterleaving, the discrete-time received signal can be expressed in the frequency domain as

$$
\mathbf{R}_{n}=\mathbf{H}_{n} \mathbf{U} \mathbf{x}_{n}+\mathbf{V}_{n}, \quad n=0,1, \cdots, N-1,
$$

where

$$
\mathbf{R}_{n}=(R[n], R[N+n], \ldots, R[(M-1) N+n])^{T}
$$

is a vector of $M$ elements which are decimated from $\mathbf{R}$ by a down-sampling factor $N$ starting from $R[n]$, the $n$th element of $\mathbf{R}$,

$$
\mathbf{H}_{n}=\operatorname{diag}(H[n], H[N+n], \ldots, H[(M-1) N+n])
$$

is an $M \times M$ diagonal matrix with diagonal elements decimated from the channel discrete frequency response (the $M N$-point discrete Fourier transform of $h[i]$ ) $H[k]$, $k=0,1, \cdots, M N-1$, by a down-sampling factor $N$ starting from $H[n]$, and $\mathbf{V}_{n}$ is a zero-mean Gaussian noise vector with covariance matrix $E\left\{\mathbf{V}_{n} \mathbf{V}_{n}^{\prime}\right\}=\sigma_{V}^{2} \mathbf{I}$, where $E\{\cdot\}$ denotes ensemble average. It is also assumed that the data symbols in $\mathbf{x}_{n}$ are mutually independent with average signal power $\sigma_{x}^{2}$ so that $E\left\{\mathbf{x}_{n} \mathbf{x}_{n}^{\prime}\right\}=\sigma_{x}^{2} \mathbf{I}$.

Finally, to recover each transmitted data vector $\mathbf{x}_{n}$, equalization and detection must be performed on each received signal vector $\mathbf{R}_{n}$. Denoting the estimated data vectors as $\hat{\mathbf{x}}_{n}$, the output data sequence $\hat{x}[i], i=0,1, \cdots, M N-1$, is then obtained from vector $\hat{\mathbf{x}}=\left(\begin{array}{llll}\hat{\mathbf{x}}_{0}{ }^{T} & \hat{\mathbf{x}}_{1}{ }^{T} & \cdots & \hat{\mathbf{x}}_{N-1}{ }^{T}\end{array}\right)^{T}$ after P/S.

Regarding the frequency-selective multipath fading channel, we use a normalized tapped delay line model and assume a full multipath diversity of order $L$. That is, all channel tap coefficients $h[i], i=0,1, \cdots, L-1$, are independent and identically distributed (i.i.d.) complex Gaussian random variables with zero-mean and variance $1 / L$.

\section{BERs OVER Multipath FAding ChanNels}

\section{A. BER Lower Bound of ML Detection}

To set up a benchmark for performance comparison, we first give a BER lower bound using the ML detection.

Assuming perfect knowledge of the channel at the receiver, the ML estimate of the $n$th data vector $\mathbf{x}_{n}$ can be obtained by minimizing the quantity

$$
\left(\mathbf{R}_{n}-\mathbf{H}_{n} \mathbf{U} \hat{\mathbf{x}}_{n}\right)^{\prime}\left(\mathbf{R}_{n}-\mathbf{H}_{n} \mathbf{U} \hat{\mathbf{x}}_{n}\right), \quad n=0,1, \cdots, N-1,
$$

through exhaust search from all possible date vectors $\hat{\mathbf{x}}_{n}$. Following a well established procedure, a lower bound of the average BER for the ML detection over frequency-selective multipath fading channels is given by 


$$
P_{e}^{m l}(M, L)=E_{h}\left\{\frac{1}{N} \sum_{n=0}^{N-1} Q\left(\sqrt{\gamma_{i n} \frac{1}{M} \sum_{l=0}^{M-1}|H[l N+n]|^{2}}\right)\right\}
$$

where $E_{h}\{\cdot\}$ denotes the ensemble average over all channel coefficients $h[i], i=0,1, \cdots, L-1$, the Q-function is defined as $Q(x)=\frac{1}{\sqrt{2 \pi}} \int_{x}^{\infty} e^{-\frac{t^{2}}{2}} d t$, and $\gamma_{i n}=\frac{\sigma_{x}^{2}}{\sigma_{V}^{2}}$ is the input SNR. To describe the impact of the data group size $M$ and the multipath length $L$ on the detection performance, we have denoted the BER lower bound (6) as a function of $M$ and $L$.

\section{B. BER of Linear Equalization}

Linear equalization is preferable in practice since it can simply use a one-tap equalizer for each subcarrier in the frequency domain. Let $C[k]$ denote the one-tap equalizer coefficient to be applied to $R[k]$ on subcarrier $k$ and

$$
\mathbf{C}_{n}=\operatorname{diag}(C[n], C[N+n], \ldots, C[(M-1) N+n])
$$

denote an $M \times M$ diagonal matrix with diagonal elements $C[l N+n], \quad l=0,1, \cdots, M-1$. The linear equalization and detection process can be described as follows. First, applying $\mathbf{C}_{n}$ to $\mathbf{R}_{n}$ produces an equalized vector $\mathbf{C}_{n} \mathbf{R}_{n}$. Second, using $\mathbf{U}^{\prime}$ to remove precoding yields the decision variable vector $\mathbf{d}_{n}=\mathbf{U}^{\prime} \mathbf{C}_{n} \mathbf{R}_{n}$. Finally, an estimate of the transmitted data vector $\mathbf{x}_{n}$ is obtained after hard decision on $\mathbf{d}_{n}$.

When the MMSE criterion is used, i.e., designing $\mathbf{C}_{n}$ so that the mean squared error (MSE) between $\mathbf{d}_{n}$ and $\mathbf{x}_{n}$

$$
\varepsilon_{n}^{2}=E\left\{\left(\mathbf{d}_{n}-\mathbf{x}_{n}\right)^{\prime}\left(\mathbf{d}_{n}-\mathbf{x}_{n}\right)\right\}
$$

is minimized, the diagonal element in $\mathbf{C}_{n}$ is found to be

$$
C[l N+n]=\frac{H^{*}[l N+n]}{\mid H[l N+n]^{2}+\frac{1}{\gamma_{i n}}}
$$

and the average BER can be evaluated as

$$
P_{e}^{m m s e}(M, L)=E_{h}\left\{\frac{1}{N} \sum_{n=0}^{N-1} Q\left(\sqrt{\frac{1}{J_{n}^{(1)}\left(\gamma_{i n}\right)}-1}\right)\right\}
$$

where

$$
J_{n}^{(1)}\left(\gamma_{i n}\right)=\frac{1}{M} \sum_{l=0}^{M-1} \frac{1}{\gamma_{i n} \mid H[l N+n]^{2}+1}
$$

is the normalized minimum MSE [5].

As seen from (9), the MMSE equalization requires the knowledge of the input SNR. If this knowledge is not available, the ZF equalization can be performed by assuming no noise is present at the receiver and selecting the equalizer coefficients $C[l N+n]=\frac{1}{H[l N+n]}$ to force the ISI to zero. However, this ZF equalization enhances noise power when the input SNR is low and causes dividing-by-zero problem at null subcarriers. In practice, we can design the linear equalizer coefficients according to a predetermined or estimated reference SNR $\gamma_{\text {ref }}$ as

$$
C[l N+n]=\frac{H^{*}[l N+n]}{\mid H\left[l N+\left.n\right|^{2}+\frac{1}{\gamma_{\text {ref }}}\right.} .
$$

Apparently, when $\gamma_{\text {ref }}=\gamma_{\text {in }}$ this linear equalization becomes the MMSE equalization, and as $\gamma_{\text {ref }} \rightarrow \infty$ it becomes the ZF equalization. Using the equalizer coefficients defined in (12), the average BER for the linear equalization can be derived as

$$
P_{e}(M, L)=E_{h}\left\{\frac{1}{N} \sum_{n=0}^{N-1} Q\left(\sqrt{\gamma_{n}(M, L)}\right)\right\}
$$

where

$$
\frac{\gamma_{n}(M, L)=}{\left[1-J_{n}^{(1)}\left(\gamma_{\text {ref }}\right)\right]^{2}}
$$

is the output SNR in the decision variable and

$$
J_{n}^{(2)}\left(\gamma_{\text {ref }}\right)=\frac{1}{M} \sum_{l=0}^{M-1} \frac{1}{\left(\gamma_{\text {ref }} \mid H[l N+n]^{2}+1\right)^{2}} .
$$

\section{AsYMPTOTICAL BERs}

To show the relationship between the system performance and the group size as well as the relationship between the system performance and the channel diversity order, let's work out two sets of asymptotical BERs for the linear equalization by asymptotical analysis. Here, the word "asymptotical" refers to the conditions when some system parameters take on extreme values. The first set represents the BERs for different block size $M$ when $L \rightarrow \infty$. The second set indicates the BERs for a given number of channel multipath $L$ (referred to as multipath diversity order) with sufficiently large data block size, i.e., $M \rightarrow \infty$. When both $M$ and $L$ approach infinity, the asymptotical BER will demonstrate the best diversity performance that the linear equalization could achieve.

\section{A. Asymptotical BER lower bounds for ML Detection}

For comparison purpose, we first work out the asymptotical BER lower bounds for the ML detection. To determine the asymptotical BER lower bounds under condition $L \rightarrow \infty$, we assume that the channel provides full multipath diversity, i.e., $L=M N$. Since $H[l N+n]=\sum_{i=0}^{M N-1} h[i] e^{-j \frac{2 \pi}{M N}(I N+n) i}$, we have

$$
\begin{aligned}
& E\left\{H[l N+n] H^{*}\left[l^{\prime} N+n\right]\right\} \\
& =\sum_{i=0}^{M N-1} \sum_{i^{\prime}=0}^{M N-1} E\left\{h[i] h^{*}\left[i^{\prime}\right] e^{-j \frac{2 \pi}{M N}(l N+n) i} e^{j \frac{2 \pi}{M N}\left(l^{\prime} N+n\right) i^{\prime}}\right. \\
& =\frac{1}{M N} \sum_{i=0}^{M N-1} e^{-j \frac{2 \pi}{M}\left(l-l^{\prime}\right) i}=\left\{\begin{array}{cc}
1, & l=l^{\prime} \\
0, & \text { otherwise }
\end{array}\right.
\end{aligned}
$$

This means that $H[l N+n]$ becomes an independent complex 
Gaussian variable with unit variance when the channel has full diversity. Then, the ensemble average in (6) can be performed on $H[l N+n]$ directly instead of $h[i]$. Denoting $H[l N+n]$ as $\alpha_{n}(l)$ for convenience, the asymptotical BER lower bound can be evaluated as

$$
\begin{aligned}
& P_{e}^{m l}(M, \infty)=\left.P_{e}^{m l}(M, M N)\right|_{N \rightarrow \infty} \\
= & \left.\frac{1}{N} \sum_{n=0}^{N-1} E_{\alpha_{n}}\left\{Q\left(\sqrt{\gamma_{i n} \frac{1}{M} \sum_{l=0}^{M-1}\left|\alpha_{n}(l)\right|^{2}}\right)\right\}\right|_{N \rightarrow \infty} \\
= & E_{\alpha}\left\{Q\left(\sqrt{\gamma_{i n} \frac{1}{M} \sum_{l=0}^{M-1}|\alpha(l)|^{2}}\right)\right\}
\end{aligned}
$$

which is the BER with diversity order $M$ (note that the subscript $n$ in $\alpha_{n}(l)$ is ignored since the ensemble averages $E_{\alpha_{n}}\left\{Q\left(\sqrt{\gamma_{i n} \frac{1}{M} \sum_{l=0}^{M-1}\left|\alpha_{n}(l)\right|^{2}}\right)\right\}$ are the same for all $\left.n\right)$. Thus, it is proved that given a group size $M$ the ML detection can only achieve diversity order $M$ even if the channel has unlimited diversity.

The second set of asymptotical BER lower bounds indicates the best performance for a given multipath diversity order $L$ but with sufficiently large data block size $M$. In this case, we have $H[l N+n]=\sum_{i=0}^{L-1} h[i] e^{-j \frac{2 \pi}{M N}(I N+n) i}$, and thus

$$
\begin{aligned}
& \sum_{l=0}^{M-1} \mid H[l N+n]^{2}=\sum_{l=0}^{M-1} \sum_{i=0}^{L-1} h[i] e^{-j \frac{2 \pi}{M N}(I N+n) i} \sum_{i=0}^{L-1} h^{*}\left[i^{\prime}\right] e^{j \frac{2 \pi}{M N}(I N+n) i^{\prime}} \\
& =\sum_{i=0}^{L-1} \sum_{i^{\prime}=0}^{L-1} h[i] h^{*}\left[i^{\prime}\right] e^{-j \frac{2 \pi}{M N}\left(i-i^{\prime}\right)} \sum_{l=0}^{M-1} e^{-j \frac{2 \pi}{M}\left(i i^{\prime}\right)} \\
& =M \sum_{i=0}^{L-1} \mid h[i]^{2}, \quad \text { for } \quad M \geq L .
\end{aligned}
$$

As $M \rightarrow \infty$, we have

$$
\begin{aligned}
& P_{e}^{m l}(\infty, L)=\left.P_{e}^{m l}(M, L)\right|_{M \rightarrow \infty}=\frac{1}{N} \sum_{n=0}^{N-1} E_{h}\left\{Q\left(\sqrt{\gamma_{i n} \sum_{i=0}^{L-1}|h(i)|^{2}}\right)\right\} \\
& =E_{h}\left\{Q\left(\sqrt{\gamma_{i n} \sum_{i=0}^{L-1}|h(i)|^{2}}\right)\right\}
\end{aligned}
$$

which is the BER with diversity order $L$.

When both $M \rightarrow \infty$ and $L \rightarrow \infty$, we have $\frac{1}{M} \sum_{l=0}^{M-1}|\alpha(l)|^{2}$ $\rightarrow 1$ and $\sum_{i=0}^{L-1}|h(i)|^{2} \rightarrow 1$. Therefore, from either (17) or (19) the asymptotical BER lower bound of the ML detection becomes

$$
P_{e}^{m l}(\infty, \infty)=Q\left(\sqrt{\gamma_{\text {in }}}\right)
$$

which is best performance a precoded OFDM system could achieve. We refer to it as the maximum diversity performance. It is the same as the BER in Gaussian channel without fading.

\section{B. Asymptotical BERs for Linear Equalization}

Following the same procedure as that we used for deriving (17), the first set of the asymptotical BERs (i.e., $L \rightarrow \infty$ ) can be expressed as $P_{e}(M, \infty)=E_{\alpha}\{Q(\sqrt{\gamma(M, \infty)})\}$ where $\gamma(M, \infty)$ has the same expression as (14) but the subscript $n$ is ignored and

$$
\begin{aligned}
& J^{(1)}\left(\gamma_{\text {ref }}\right)=\frac{1}{M} \sum_{l=0}^{M-1} \frac{1}{\gamma_{\text {ref }}|\alpha(l)|^{2}+1} \\
& J^{(2)}\left(\gamma_{\text {ref }}\right)=\frac{1}{M} \sum_{l=0}^{M-1} \frac{1}{\left(\gamma_{\text {ref }}|\alpha(l)|^{2}+1\right)^{2}} .
\end{aligned}
$$

The second set of the asymptotical BERs is reached as $M \rightarrow \infty$ under a given multipath diversity order $L$, and can be expressed as $P_{e}(\infty, L)=E_{h}\{Q(\sqrt{\gamma(\infty, L)})\}$ where $\gamma(\infty, L)$ has the same expression as (14) but

$$
\begin{aligned}
& J^{(1)}\left(\gamma_{\text {ref }}\right)=\frac{1}{2 \pi} \int_{0}^{2 \pi} \frac{1}{\gamma_{\text {ref }}\left|H\left(e^{j \omega}\right)\right|^{2}+1} d \omega \\
& J^{(2)}\left(\gamma_{\text {ref }}\right)=\frac{1}{2 \pi} \int_{0}^{2 \pi} \frac{1}{\left(\gamma_{\text {ref }} \mid H\left(e^{j \omega}\right)^{2}+1\right)^{2}} d \omega
\end{aligned}
$$

where $H\left(e^{j \omega}\right)$ is the Fourier transform of $h[i]$.

When both $M \rightarrow \infty$ and $L \rightarrow \infty$, a closed-form expression of the best performance that the linear equalization could offer can be derived as $P_{e}(\infty, \infty)=Q(\sqrt{\gamma(\infty, \infty)})$, where $\gamma(\infty, \infty)$ has the same expression as (14) but

$$
\begin{aligned}
& J^{(1)}\left(\gamma_{\text {ref }}\right)=\int_{0}^{\infty} \frac{e^{-\rho}}{\gamma_{\text {ref }} \rho+1} d \rho \\
& J^{(2)}\left(\gamma_{\text {ref }}\right)=\int_{0}^{\infty} \frac{e^{-\rho}}{\left(\gamma_{\text {ref }} \rho+1\right)^{2}} d \rho
\end{aligned}
$$

which can be derived from (21) and (22) respectively. For example, to derive (25), we notice that $|\alpha(l)|^{2}$ in (21) is chisquare-distributed with two degrees of freedom and probability distribution function (pdf) $e^{-\rho}$. As $M \rightarrow \infty$, the average $\frac{1}{M} \sum_{l=0}^{M-1} \frac{1}{\gamma_{r e f}|\alpha(l)|^{2}+1}$ over $l$ can be replaced by ensemble average $\int_{0}^{\infty} \frac{e^{-\rho}}{\gamma_{i n} \rho+1} d \rho .^{\dagger}$

\section{Asymptotical BER AND SNR DEgRADATION}

Comparing the maximum diversity performance of the ML detection with the asymptotical BERs of the linear equalization derived above, we are now able to gain a full understanding of the diversity performance of the linear equalization. Fig. 2 shows the $P_{e}(\infty, \infty)$ curves as functions of $E_{b} / N_{0}$, by replacing the input SNR $\gamma_{i n}=2 E_{b} / N_{0}$ for QPSK, where $E_{b}$

\footnotetext{
${ }^{\dagger}$ Defining a special function $E^{(n)}(x)=\int_{x}^{\infty} \frac{e^{-t}}{t^{n}} d t,(25)$ and (26) can be alternatively evaluated by $J^{(n)}(\gamma)=\frac{1}{\gamma^{n}} e^{\frac{1}{\gamma}} E^{(n)}\left(\frac{1}{\gamma}\right)$ for $n=1$ and 2 .
} 
is the signal energy per bit and $N_{0}$ is the noise power spectral density. The reference normalized SNR is set to $\left(E_{b} / N_{0}\right)_{\text {ref }}=$ $0,10,20,30,40 \mathrm{~dB}$ respectively. $P_{e}^{m l}(\infty, \infty)$ and $P_{e}^{m m s e}(\infty, \infty)$, which is the performance lower bound of the linear equalization, are also displayed for comparison purpose.

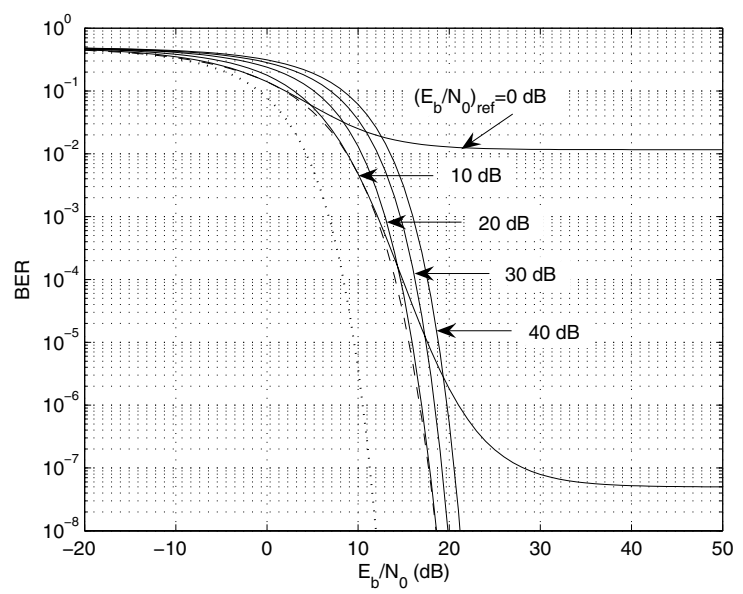

Fig. 2. Asymptotical BERs of precoded OFDM with linear equalization under different reference normalized SNRs (solid lines). The asymptotical BERs for ML detection (doted line) and MMSE equalization (dashed line) are also displayed.

As we know, the diversity order describes how fast the BER decreases as the SNR increases, i.e., it is related to the slope of the BER curve. From Fig. 2 we see that a BER curve of the linear equalization for a given reference SNR has similar slope to that of the maximum diversity BER curve for SNRs below the reference SNR. As the SNR increases beyond the reference SNR, the BER curve gradually goes flat. This observation indicates that the linear equalization achieves the same diversity order as the ML detection for SNRs lower than the reference SNR (though the BER itself is worse than that of the ML detection) but the diversity is lost when the SNR is higher than the reference SNR. We refer to the SNRs below the reference SNR as the operational SNR range.

Furthermore, we notice that the asymptotical BERs $P_{e}^{m l}(\infty, \infty)$ and $P_{e}(\infty, \infty)$ are evaluated by the same Q-function but with SNR $\gamma_{\text {in }}$ and $\gamma(\infty, \infty)$ respectively. Thus the performance degradation can be more efficiently described by the SNR degradation. We call this SNR degradation as the asymptotical SNR degradation, which is defined as

$$
\begin{aligned}
D & \equiv-10 \log \left(\frac{\gamma^{(\infty, \infty)}}{\gamma_{\text {in }}}\right) \\
& =10 \log \left(\frac{\gamma_{\text {in }}\left[1-J^{(1)}\left(\gamma_{\text {ref }}\right)\right] J^{(1)}\left(\gamma_{\text {ref }}\right)+\left[J^{(1)}\left(\gamma_{\text {ref }}\right)-J^{(2)}\left(\gamma_{\text {ref }}\right)\right)\left(\gamma_{\text {ref }}-\gamma_{\text {in }}\right)}{\left[1-J^{(1)}\left(\gamma_{\text {ref }}\right)\right]^{2}}\right) .
\end{aligned}
$$

Fig. 3 shows the asymptotical SNR degradation as a function of the normalized SNR $E_{b} / N_{0}$ with $\left(E_{b} / N_{0}\right)_{\text {ref }}=0$, $10,20,30,40 \mathrm{~dB}$ respectively. We see that when the SNR is lower than the reference SNR the degradation is almost a constant, which implies the same slope on the BER curve. Once the SNR goes beyond the operational range, the degradation increases rapidly, which indicates the loss of diversity. As the reference SNR increases, the operational range becomes wider but the SNR degradation increases too.

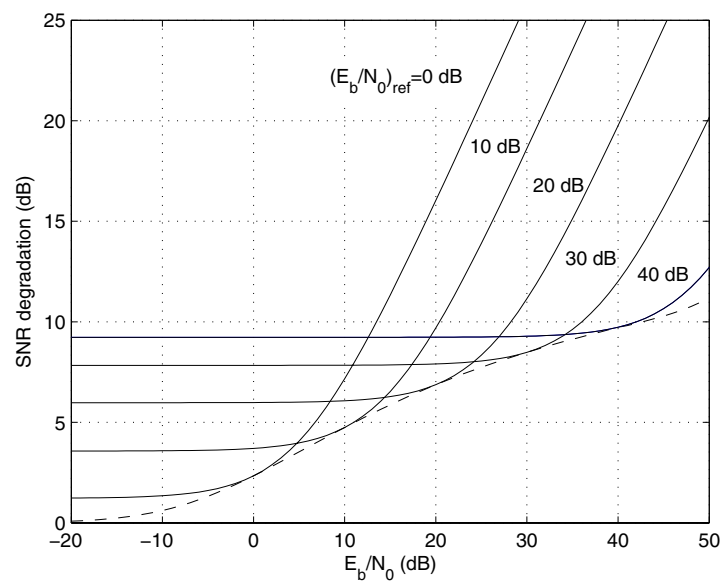

Fig. 3. Asymptotical SNR degradations for linear equalization under different reference normalized SNRs (solid lines). The asymptotical SNR degradation for MMSE equalization (dashed line) represents a lower bound.

\section{CONCLUSIONS}

We have shown that a practical linear equalization achieves the maximum multipath diversity for the SNRs within an operational range which is determined by the reference SNR used to design the linear equalization coefficients. However, once the SNR goes across the operational threshold (i.e., the reference SNR), the diversity advantage is lost. If the SNR is known at the receiver, the MMSE equalization will offer the lower bound performance. The derived closed-form asymptotical BER and SNR degradation expressions not only provide an efficient way to evaluate the potential performance that the linear equalization could achieve but also serve as a useful tool for optimal receiver design.

\section{REFERENCES}

[1] Z. Wang and G. B. Giannakis, "Linearly precoded or coded OFDM against wireless channel fades?," in Proceedings of Signal Processing Advances in Wireless Communications Workshop, Taoyuan, Taiwan, March 20-23, 2001, pp. 267-270.

[2] Z. Liu, Y. Xin, and G. B. Giannakis, "Linear constellation precoding for OFDM with maximum multipath diversity and coding gains," IEEE Transactions on Communications, Vol. 51, No. 3, March 2003, pp. 416427.

[3] C. Tepedelenlioglu, "Maximum multipath diversity with linear equalization in precoded OFDM systems," IEEE Transactions on Information Theory, Vol. 50, No. 1, January 2004, pp. 232-235.

[4] M. L. McCloud, "Analysis and design of short block OFDM spreading matrices for use on multipath fading channels," IEEE Transactions on Communications, Vol. 53, No. 4, April 2005, pp. 656-665.

[5] X. Huang, "Diversity performance of precoded OFDM with MMSE equalization," presented at the 2007 International Symposium on Communications and Information Technologies (ISCIT2007), Sydney, Australia, 16 -19 October, 2007. 